\title{
Prostatakarzinom: Das Risiko für Thromboembolien reduzieren
}

\author{
Venöse Thromboembolien gehören zu den gefürchteten Komplikationen \\ maligner Erkrankungen, treten aber auch als Folge verschiedener chronischer \\ Erkrankungen auf. Bisher war unklar, ob komorbide Patienten mit einem \\ Prostatakarzinom unter einem höheren Thromboembolierisiko leiden als \\ Männer mit ähnlichen Komorbiditäten ohne diese maligne Erkrankung.
}

n der Allgemeinbevölkerung gehen venöse Thromboembolien (VTE) mit einer Häufigkeit von etwa 1:1.000 bei steigender Inzidenz mit fortschreitendem Lebensalter einher. Höheres Alter gilt auch als wichtigster Risikofaktor für das Prostatakarzinom (PCa). Schätzungen zufolge leiden etwa $40 \%$ der Männer zum Zeitpunkt der Diagnosestellung eines $\mathrm{PCa}$ unter chronischen Begleiterkrankungen. Dank der Fortschritte in der Therapie versterben heute viele Patienten eher an den Folgen ihrer Komorbiditäten als an der malignen Erkrankung selbst.

Die Klärung der Frage nach möglichen Interaktionen zwischen dem PCa und Begleiterkrankungen könnte zu präventiven Behandlungsstrategien und zur Prognoseverbesserung beitragen. Dänische Wissenschaftler führten dazu eine landesweite Datenbank-basierte Kohortenstudie mit allen 44.035 zwischen 1995 und 2011 registrierten PCa-
Fällen durch und „matchten“ sie statistisch mit 231.810 Männern aus der Normalbevölkerung nach Alter und Begleiterkrankungen.

Insgesamt entwickelten 894 PCa-Patienten und 2.360 Männer ohne maligne

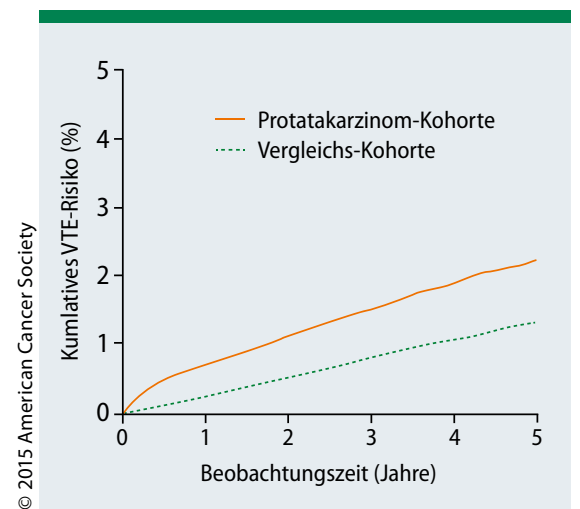

Abb. 1: Das kumulative VTE-Risiko ist bei Prostatakarzinompatienten über einen 5-Jahres-Zeitraum deutlich erhöht.
Prostataerkrankung innerhalb von 5 Jahren eine VTE. Somit war das VTERisiko in der PCa-Gruppe mit 2,2\% gegenüber dem Vergleichsarm $(1,3 \%)$ deutlich erhöht (Abb. 1).

Die standardisierte 1-Jahres-Rate für das Neuauftreten einer VTE betrug für multimorbide PCa-Patienten 15/1.000 Personenjahre.

Als Hochrisikofaktoren erwiesen sich in dieser Untersuchung höheres Lebensalter, metastasierte Erkrankung, hoher Gleason-Score, Hochrisiko-PCa nach d'Amico und ein vorangegangener chirurgischer Eingriff.

Fazit: Diese Daten legen eine klinisch relevante Interaktion zwischen einem manifesten Prostatakarzinom, hohem Komorbiditätsgrad und in der Folge einem erhöhten Risiko für venöse Thromboembolien (VTE) nahe. Präventive Maßnahmen zur Reduzierung des Thromboembolierisikos könnten als Ansatz dienen, die Prognose von Patienten mit Prostatakrebs zu verbessern.

Wolfgang Zimmermann

Ording AG et al. Prostate cancer, comorbidity, and the risk of venous thromboembolism: a cohort study of 44.035 Danish prostate cancer patients, 1995-2011. Cancer. 2015;121(20):3692-9.

\section{Nodal positives Prostatakarzinom: ADT mit oder ohne Radiotherapie?}

\section{Das Management des klinisch nodal positiven ( $\mathrm{cN}+$ ) Prostatakarzinoms (PCa) bleibt kontrovers aufgrund der beschränkten Datenlage und mangels robuster Studien mit Therapieempfehlungen für die Praxis. Was leisten Radiotherapie plus Androgenentzug für diese Erkrankung?}

$B_{d}^{a}$ asis einer Analyse hierzu waren Daten der US-amerikanischen „National Cancer Data Base“ von 3.540 Patienten mit $\mathrm{cN}+\mathrm{PCa}$ ohne Fernmetastasen. Verglichen wurden die Überlebensraten nach ADT („androgen deprivation therapy“) sowie ADT plus Radiotherapie (RT). In der Untergruppe, die zwischen 2004 und 2006 diagnostiziert und mit ADT bzw. ADT/RT behandelt worden war, hatten sich $32 \%$ der Patienten einer
ADT, 51,4\% einer ADT und RT unterzogen. Nach dem Matching verblieben in jeder Gruppe 318 Patienten. Die Analyse ergab eine Reduktion der 5-Jahres-Mortalität aufgrund aller Ursachen um $50 \%$ für Patienten mit nodal positivem Prostatakarzinom, die zusätzlich zur ADT radiotherapeutisch behandelt wurden. Zudem legen diese Daten nahe, dass etwa die Hälfte der Patienten mit dieser Tumorkondition nicht die optimale The- rapie mit dem Ziel einer dauerhaften Krankheitskontrolle erhalten hatte.

Fazit: Patienten mit klinisch nodal positivem Prostatakarzinom profitieren von einem um $50 \%$ verbesserten 5 -Jahresüberleben, wenn sie zusätzlich zur ADT auch eine Radiotherapie erhalten. Dieser, auf Basis einer großen Population nachgewiesene, statistisch signifikante Überlebensvorteil sollte Anlass geben für eine Reevaluation gegenwärtiger Praxisleitlinien.

Wolfgang Zimmermann

Lin CC et al. Androgen deprivation with or without radiation therapy for clinically node-positive prostate cancer. J Natl Cancer Inst. 2015; 107(7). pii: djv119. 